\title{
Infancia y adolescencia de un traductor latinoamericano, o acerca de la formación de José Coronel Urtecho
}

SERGIO RAIMONDI Universidad Nacional del Sur, Argentina / sr.raimondi@gmail.com

\section{Resumen}

¿Cómo se forma un traductor? ¿Cuál es su vínculo con otras lenguas además de la materna? ¿Hay relación entre el aprendizaje de esas lenguas y el aprendizaje de sus literaturas? ¿Qué lengua se aprende cuando se aprende una lengua? Preguntas como estas emergen de la lectura de la crónica «El americanismo en la casa de mi abuelo» (1962), en la que el nicaragüense José Coronel Urtecho deja entrever su "destino-de-traducción» en los años previos a su viaje a San Francisco, Estados Unidos, en 1924.

Palabras clave: traducción / José Coronel Urtecho / poesía latinoamericana / poesía norteamericana modernista / literaturas comparadas

\section{Childhood and adolescence of a Latin American translator, or about José Coronel Urtecho's training}

Abstract

How does someone become a translator? What is his bond to languages other than the mother tongue? Is there a relationship between learning those languages and learning their literatures? Which language is learned when a language is learned? Questions like these emerge from the reading of the chronicle «El americanismo en la casa de mi abuelo» (1962), in which it is possible to see how the «destiny-of-translation» of the Nicaraguan José Coronel Urtecho occurs in the years prior to his trip to San Francisco, US, in 1924.

Key words: translation studies / José Coronel Urtecho / latin-american poetry / modernist american poetry / comparative literatures

Recibido: 30/5/2019. Aceptado: 14/6/2019

Para citar este artículo: Raimondi, S. (2019). Infancia y adolescencia de un traductor latinoamericano, o acerca de la formación de José Coronel Urtecho. El taco en la brea, 10 (junio-noviembre), 93-102. Santa Fe, Argentina: UNL. DOI: 10.14409/tb.v1i10.8689 
Apenas un año antes de la aparición de Antología de la poesía norteamericana en editorial Aguilar en 1963, trabajo que — con la colaboración de Ernesto Cardenal - ampliaría su primera gran obra de traducción de 1949 y lo consolidaría como introductor de la poesía modernista norteamericana en Nicaragua y uno de sus difusores fundamentales en América Latina, José Coronel Urtecho publica una crónica sobre sus años de infancia y adolescencia. Titulada «El americanismo en la casa de mi abuelo», esa narrativa, extendida entre 1910 y 1924 (es decir, entre sus cuatro y sus dieciocho años), ocupa el tiempo exacto previo a su decisión de viajar a los Estados Unidos, donde tomaría contacto y comenzaría a traducir la poesía modernista allá emergente. Si bien a lo largo de la crónica no hay ninguna referencia explícita a su trabajo como traductor, el hecho de que quien la escribe sea un Urtecho ya consolidado como tal permite pensar estos «recuerdos» y reflexiones sobre su período infantil y adolescente como el tipo de materiales que Antoine Berman considera adecuados para rastrear un «destino-de-traducción»: no sólo la trayectoria que hace de un traductor un traductor sino, más específica y sutilmente aun, el impulso que lo lleva a traducir.' Desde esa perspectiva, la crónica admite preguntas sobre la relación del niño y adolescente Urtecho con otras lenguas además del español; sobre los modos singulares de esos aprendizajes; sobre sus conceptualizaciones en torno a las literaturas francesa, inglesa y norteamericana y, también, sobre el rol que cumple, en esa formación, la lectura de traducciones. Pero antes de avanzar en estas inquietudes conviene detenerse en el inicio mismo de la crónica, el cual advierte sobre la necesidad de no perder de vista, al abordar cuestiones más generales, la situación particular de un lector y traductor en esta parte del mundo.

Que el primer recuerdo que proponga en su crónica el ya consolidado traductor de poesía norteamericana en relación con los Estados Unidos sea no ya su propia imagen a los cuatro años con una blusa de marinero que exhibe, bordadas en seda, las banderas norteamericana y nicaragüense entrelazadas sino la «espantosa impresión» de su padre apareciendo con «unas tijeras descomunales» para recortar «con increíble celeridad, de un solo tijeretazo» (1962:25) la bandera del país más al norte, habilita una primera advertencia: la relación de Urtecho con aquella nación no puede ser concebida sino desde la tensión. La primera formulación de esa tensión es, evidentemente, política: el padre expone con su gesto la perspectiva de un liberal preocupado por la doctrina del «destino manifiesto» norteamericano, radicalizado por entonces con la anexión de los territorios mexicanos, la invasión a Filipinas y la actitud más que amenazante sobre la propia Nicaragua. Allá por la década del 10 del siglo pasado, recuerda Urtecho, «Hablar de los Estados Unidos era hablar de política» (26); y hablar de política era discutir en una escalada gradual de agresiones: no había más opción que «tomar partido en pro o en contra» (26). La solución que encontrará Urtecho a través del tiempo consistirá en separar lo más netamente posible los ámbitos de la política y la literatura, pensados como series heterogéneas: «lo curioso es que no me gustan los Estados Unidos, o por lo menos no he podido lograr hasta ahora que me guste de veras la realidad norteamericana, si no es vista a través de su literatura» (30). Esa aproximación doble persistirá a lo largo de su vida: «No sé si he superado esa ambivalencia» (26). La escena inicial, o iniciática, con su padre es entonces una declaración de principios. ¿Será posible recortar la escena del recorte de la bandera de un posible «destino-de-traducción» que tendrá en la poesía norteamericana su objeto de preferencia? En principio, la escena advierte que la distinción de ese objeto de preferencia no puede distraer la posición de un traductor en América Latina y en Centroamérica en particular. También advierte que, previo a su primer contacto con el inglés, ese niño ya sabe, con mayor o menor conciencia, que esa lengua está tramada por la violencia. 
Pero si en 1910 «Hablar de los Estados Unidos era hablar de política», ¿qué se podría decir en 1962 - apenas tres años después de la revolución cubana—, cuando Urtecho publica esta crónica en la que elige la escena paterna como rito iniciático? Urtecho reconoce la nueva situación coyuntural; de hecho, tras aclarar su necesidad de separar la política norteamericana de la literatura norteamericana, y también de mencionar su desagrado general por aquel país, agrega que, de no haber sido por su fascinación por los poetas, «tal vez [hubiera] terminado sintiendo por los Estados Unidos lo mismo que mi padre, como hoy no es raro entre intelectuales latinoamericanos» (31). El comentario señala no solo su distinción con respecto a la percepción de su progenitor; señala también cómo aquella tensión inicial permanece, transformada: ahora se muestra en su vínculo con una «familia» más vasta, en este caso la de los «intelectuales» del continente, con la que, como si aún estuviera dispuesto a vestir la casaca con las dos banderas entrelazadas, Urtecho toma prudente distancia. La forma de esa distancia sigue basada en su convicción de la diferencia cualitativamente heterogénea entre la política y la literatura, pero en un momento en que optar por esa diferenciación podía resultar de una sutileza inverosímil. Porque también en los inicios de los 60 en América Latina hablar de los Estados Unidos, aunque se tratara de su literatura, era por entonces «hablar de política». Por eso en esa mención sobre su diferencia con respecto a los «intelectuales latinoamericanos» exhibe que, ya con más de treinta años dedicados a la traducción de poesía norteamericana, la escena de violencia vivida con su padre forma parte constitutiva de su misma auto-figuración. A diferencia de la posición de indefensión que tenía a los cuatro años, ahora asume la ofensiva: «No tengo empacho en confesar que a mí me gusta más la literatura norteamericana que la nuestra de América Latina, y que en muchos aspectos la considero francamente superior a esta» (30).

Muerto su padre liberal, el futuro traductor pasa a vivir en la casa del abuelo materno. Esa casa está sostenida sobre tres adjetivos definitivos: «una casa tradicional, profundamente conservadora, donde aún se conservaban la mayoría de las costumbres coloniales». Todo cambia en esa casa en relación con la percepción de los Estados Unidos. Ahí todos eran «americanistas»: era un «ambiente de admiración y hasta de culto a todo lo "americano"» (25). En esa casa encontrará algo más que una bandera norteamericana: encuentra el inglés. En realidad, empieza a habitar un espacio donde el español ya no es la única lengua. Al menos tres lenguas se hablan en esa casa de su infancia y adolescencia: español, francés e inglés. Así, antes de aprender el francés o el inglés, el futuro traductor aprende algo más relevante: que no existe una lengua. También aprende que ninguna lengua se aprende; del todo, al menos. El francés por ejemplo lo aprende en parte a leer, con «mayor gusto» que el español, porque — según dice — nadie lo obliga a hacerlo. Pero no lo aprende a hablar; de hecho, su escucha implicaba invariablemente una comprensión a medias. Fragmentos de «Le Lac» $\mathrm{y}$ «El crucifijo» de Lamartine se escuchaban en la voz de la madre, educada en Francia: esos «versos románticos, de una armonía inmensamente dulce y evocadora, entendidos a medias, me abrían una ventana a un paisaje ideal, proyectado en el ámbito del sueño, envuelto en una bruma de misterio» (27). El Urtecho niño deja entender así no solo su relación incompleta con una lengua distinta del español; exhibe también su capacidad temprana para entender que, por lo menos en relación con la poesía, es posible comprender sin comprender, como si ya entonces hubiera estado dispuesto a subscribir al Benjamin que, en torno a esos mismos años, preguntaría y respondería en "La tarea del traductor»: «¿Qué "dice” una obra literaria? ¿Qué comunica? Muy poco a aquel que la comprende. Su razón de ser fundamental no es la comunicación ni la afirmación» (127). 
Tampoco el inglés se aprende del todo en aquella casa. Pero, ¿qué inglés era ese inglés? En realidad el inglés se multiplica en diferentes ingleses en esa casa de infancia. El futuro traductor aprende entonces no solo que hay más de una lengua, sino que una lengua tampoco es, exactamente, una. El inglés de los versos de Shakespeare que se recitaba en la casa no era el mismo que el inglés de sus tías educadas en los Estados Unidos; tampoco era exactamente el inglés que su abuelo practicaba todos los días, durante una hora, con un maestro jamaiquino; y tampoco el de la niñera negra que acompañó a una de sus tías radicadas en Estados Unidos en una de sus visitas a Granada. Este último inglés en particular es el único que recuerda haber aprendido el niño Urtecho: «el inglés de los negros de la Luisiana» (1962:30). De todos los que se hablaban o escuchaban en la casa de su abuelo, ese inglés ya está desvinculado del mundo europeo y socialmente inscripto en territorio norteamericano. Urtecho recuerda no solo haberlo aprendido; recuerda también haberlo olvidado al retornar aquella niñera con su tía a Nueva Orleans, al punto de que cuando en el internado, ya adolescente, le den poemas en inglés (Whitman, más exactamente), advertirá que ya no se siente capacitado, aunque no fuera «de los últimos de la clase», para «leerlos en el original» (31). Urtecho proyecta así al menos tres cuestiones para sus primeros años de formación: hay más de una lengua; la idea de una lengua como entidad única y unificadora es una ficción, porque aun lo que se nombra como «una lengua» implica multiplicidad; la experiencia de adquisición de una lengua puede ser no solo incompleta, como muestra su vínculo con el francés, sino intermitente: se puede disponer de una lengua y se puede también olvidarla; o sea, el sentido de la posesión de una lengua no es definitivo.

Hay que detectar además una inversión en la experiencia tanto intermitente como incompleta de su relación con esas otras dos lenguas además del español. El niño Urtecho aprende a leer el francés, aunque no a hablarlo, y aprende a hablar el inglés, aunque no a leerlo. ¿No está dando esa inversión a entender que aprende a leer el francés porque en esa lengua hay una literatura (ahí están los tomos de Hachette y Garnier de la biblioteca de su madre), y que aprende a hablar el inglés (el «americano», no el europeo, el de los negros de Luisiana), porque en esa lengua — por lo menos todavía, como experimenta aún en la casa «americanista» de su abuelo— no la hay? Por lo pronto conviene detenerse en la cualidad particular del inglés que aprende y olvida, porque en la variante particular de esa lengua ya aparece inscripto su «destino-de-traducción». La inflexión de ese inglés «de los negros de la Luisiana» suscribe - literalmente—su fascinación por el Huckleberry Finn de Mark Twain, libro al que en Rápido tránsito (donde reúne las crónicas de sus viajes a los Estados Unidos, incluido el primero e inaugural) contrapone a Atalá de Chautebriand, uno de los tantos de la biblioteca materna. Si en el libro francés el río es "más fantasía tropical», en aquel es «la propia realidad, vivida, asimilada» (1959:86), una diferencia sustancial sostenida en razón de un estilo modelado más desde una ficción retórica del habla que desde una ficción retórica de la escritura literaria; así describe Urtecho ese estilo: «libre, sencillo, oral, con la cadencia y la estructura de las frases habladas» (87). Es decir: esa versión singular del inglés que aprende de la niñera negra de Luisiana, radicalizada por el carácter oral del aprendizaje, ofrece el horizonte adecuado para que el traductor pueda ser capaz de escuchar la lengua hablada como modelo verosímil en la literatura: no ya siquiera en los decimonónicos Twain o Whitman; también en la mismísima poesía modernista que encontrará a su llegada a los Estados Unidos a los 18 años. Tal como anota también en Rápido tránsito, la mayoría de esos poetas escribía «en un lenguaje usual, viviente, como el que se habla, con los sonidos y las cadencias de la voz humana» (59). No es solo entonces el aprendizaje de 
un inglés genérico el que lo conduce a su «destino-de-traducción», sino la variante particular del inglés que aprende, y la modalidad de ese aprendizaje que privilegia una instancia oral, al revés de lo que le sucede con el francés, aprendido para ser ejercido en la lectura literaria. El inglés enseñado por la niñera negra supone una aptitud que estará menos en el ojo que en el oído, y que le permitirá escuchar la singularidad de una poesía que propone, desde la poética inaugural de Walt Whitman, la escucha como base de todo canto: «I hear America singing».

En la insistencia con la que el cronista plantea cómo aquel que era en la infancia y la adolescencia se formaba a partir de una relación inestable y básicamente incompleta en su conocimiento de las lenguas, ya sea el francés de la madre, ya sea el inglés de la niñera negra, se puede reconocer tanto la metodología de trabajo como la forma genérica predilecta con la que resuelve su trayecto de traductor. Así, en uno de los últimos testimonios, Urtecho afirma: «Yo traduje porque esa era una manera de aprender inglés» (1993:53). La confesión amerita detención: la actividad de traducción no se ejecuta porque hay un dominio cabal de la lengua a traducir; se ejecuta justamente como un modo de aprenderla. Esa afirmación ubica a Urtecho, entonces, al margen de una concepción profesionalizada de la traducción, y ofrece el corpus amplísimo de sus traducciones (en las que nunca ofrecerá el inglés para ser cotejado junto a sus propias versiones) como testimonio de su proceso inacabado de aprendizaje lingüístico. Así continúa en «Prólogo conversado»: «Desde los Estados Unidos yo había traído un cierto bagaje de traducciones y el hábito de traducir... Yo traducía lo que era traducible para mí. Lo que no podía traducir porque me costaba lo dejaba; traducía lo que me salía» (54). El aprendizaje inestable e incompleto de la lengua afecta entonces la selección de sus objetos de traducción y justifica, por tanto, el soporte predilecto mediante el cual Urtecho presentará su trabajo una y otra vez (Panorama y antología de poesía norteamericana, 1949; El Lincoln de los poetas, 1951; Antología de poesía norteamericana, 1963; Ezra Pound, 1979): la «antología». Ninguna obra completa será el objeto de su impulso de traducción, porque no hay experiencia ninguna de compleción ni en su percepción de la lengua, ni en su percepción de las literaturas.

Porque el conocimiento de las literaturas, para el niño y adolescente Urtecho, es tan incompleto como el de las lenguas que las sostienen. ¿Cuál era para él la literatura francesa? Los clásicos de Hachette y Garnier que tenía su madre: Corneille, Racine, Moliére, La Fontaine. Y ¿cuál la británica? Obvio: Shakespeare, porque era «cosa corriente» recitarlo «entre los granadinos» cuando él era chico, además de Byron, alguna vez (1962:27). ¿Obtiene el niño conclusiones de esto? Si bien no lo dice exactamente, es evidente que comprende que las lenguas europeas como ese francés y ese inglés son lenguas de clásicos; es decir: lenguas de literaturas que ya poseen una tradición. Las mismas ediciones francesas de su madre lo confirman en su misma materialidad. Por eso es entendible que el niño Urtecho se pregunte si era posible la existencia de una literatura sostenida no ya desde el francés maternal, o el inglés europeo de los parientes que recitaban Shakespeare, sino desde ese inglés fugaz y aprendido con la niñera negra. Aun en la casa «americanista» de su abuelo, cuenta Urtecho, «casi todos tenían idea de la literatura inglesa, y apenas conocían la norteamericana» (27). La literatura que se escribía en la lengua de la niñera no disponía al parecer de clásicos ni de tradición. «A los Estados Unidos les faltaba historia» (30), recuerda Urtecho que le repetía su madre. Y ese niño pensaba entonces que «era una lástima que en los Estados Unidos no hubiera habido reyes, ni santos, ni papas, ni castillos provenzales, ni palacios de Versalles... ni catedrales de Notre Dame... ni Napoléon, ni fábulas de La Fontaine» (29-30). Tal como había escuchado alguna vez: «Estados Unidos era un pueblo bárbaro sin escritores ni poetas realmente universales» 
(29). Aun en la casa «americanista» del abuelo, los paradigmas para pensar la cultura y la literatura eran, decisivamente, europeos; más que europeos, como averiguará más tarde el propio Urtecho, franceses: esa idea de los Estados Unidos como país «nuevo» sin escritores capaces de ofrecer la «universalidad» se podía encontrar, por ejemplo, en la obra de Paul Bourget, Outre-Mer. Notes sur l'Amerique, de 1894. A posiciones de esta índole se referirá a la vuelta de su viaje de Estados Unidos, cuando publica, a la par de su «Oda a Rubén Darío», sus primeras traducciones del poeta norteamericano Carl Sandburg: «En libros rezagados que son manuales de espíritu entre nosotros se dice todavía la vieja afirmación de que los yanquis son colonos literarios de Inglaterra» (1927:2). Por eso el aprendizaje del inglés particular de la niñera negra cobra así otro sentido para su «destino-de-traducción»: habilita la sospecha de quien empieza a evaluar lo que puede ofrecer una literatura sin clásicos ni monumentos. Esa sospecha era doble. El niño y adolescente se empieza a preguntar, por un lado, si la ausencia de literatura norteamericana no será desconocimiento. Nadie por ejemplo en esa casa sabía, como se enteraría él mismo de primera mano al viajar a los Estados Unidos, «de la profunda revolución literaria de nuestro tiempo en los Estados Unidos, que tanta influencia y resonancia tendrían en el mundo moderno» (1962:29), la cual estaba sucediendo exactamente mientras el niño escuchaba que en términos literarios Estados Unidos era un vacío. Por otro, aparece la pregunta acerca de si una literatura sin clásicos y sin monumentos no constituirá menos un vacío que la oportunidad para la emergencia de la cualidad moderna por excelencia: la de lo nuevo.

Longfellow era la única referencia a la literatura norteamericana celebrada en la casa del abuelo. Se lo recitaba con frecuencia. El niño o adolescente recuerda haber oído el verso «the forgetme-nots of the angels» como una metáfora de las estrellas; recuerda también haber oído que ese verso era «el colmo de lo bello» (30). Todo el «destino-de-traducción» de Urtecho parece destinado a contrariar la poética que sostiene ese verso. La confirmación de la sospecha de que los Estados Unidos podía ofrecer una literatura «nueva» allí donde hasta entonces había Longfellow o vacío se la va a ofrecer, antes que la poesía modernista que encontrará al llegar a California, Walt Whitman. Whitman es el descubrimiento de que ese vacío podía ser en realidad «un mundo insospechado, maravillosamente nuevo, con infinitas posibilidades y prodigiosa energía, pleno de vida y alegría, juventud y esperanza» (31). Es el descubrimiento de que una poesía americana, ya no sostenida por el francés de los clásicos de Hachette, ni por el inglés de Shakespeare, era posible. Descubrir a Whitman es descubrir un clásico cuando todavía no se ha conformado como tal; es descubrir una poesía cuando todavía no ha devenido monumento. Pero es también, sobre todo y por primera vez en su trayectoria vital, la revelación de un mundo percibido en términos locales: una poesía americana que dé cuenta del mundo americano. Esa escala más amplia que la nacional está en una frase: «Me imaginaba al bardo de todo un continente» (31).

En la formación de este traductor latinoamericano, entonces, hay que contar tanto un aprendizaje incompleto de las lenguas como un aprendizaje incompleto de las literaturas. No hay, en la lengua literaria francesa que aprende el niño Urtecho, ni Baudelaire, ni Rimbaud, ni —mucho menos- Apollinaire o Cendrars. ¿Por qué? Sencillamente porque no estaban en la biblioteca de su madre. El descubrimiento de Whitman se recorta así en una singularidad excepcional frente a Lamartine, frente a Shakespeare y frente a Longfellow. Frente al «ámbito del sueño» y la «bruma del misterio» de Lamartine, o frente a «las no-me-olvides de los ángeles» de Longfellow, Whitman presentará un mundo entre comillas real donde todo se hace con gerundios y carácter 
de fundación: invadiendo, explorando, poblando, talando, arando, regando, plantando, arreando, edificando. $\mathrm{Al}$ menos tres de esos gerundios («arando", «regando", "plantando») le deben haber permitido imaginar que podía existir una poesía en la que las «no-me-olvides» estuvieran no en el cielo angelical de la noche, sino en la tierra diurna del trabajo. Urtecho estaba terminando su educación secundaria con la primera promoción del Colegio Centroamérica de Granada, regido por padres jesuitas, en 1924. El descubrimiento de Whitman fue la justificación mayor de su decisión - tan diferente a la de los granadinos de su generación— de no ir a París. Para ese adolescente de 18 años Whitman era el signo de que era posible una poesía nueva de este lado del Atlántico: «Creo que yo era el único que deseaba precisamente lo contrario, ir a los Estados Unidos a buscar lo que solo se hallaba en París, según decían los poetas: una nueva poesía, una nueva manera de ver la vida» (31).

$\mathrm{Si}$ en esos primeros años del siglo $\mathrm{XX}$, antes de su viaje y su encuentro con la emergente poesía modernista norteamericana, el orden literario que constituye el niño y adolescente Urtecho para privilegiar el impacto de las panorámicas humanas de Whitman es básicamente decimonónico, la pregunta es inevitable: ¿y Poe? Por supuesto, no es un detalle que su percepción de este poeta provenga de lecturas francesas posteriores (Baudelaire, Mallarmé) y, sobre todo, de los modernistas latinoamericanos: Darío, en particular, que brilla por su ausencia en esta crónica de sus años de infancia y adolescencia. Junto a Darío, el norteamericano Poe queda del lado de la biblioteca francesa de la madre. A diferencia de la cualidad social y territorialmente situada que encuentra el adolescente Urtecho en Whitman, a la poesía de Poe no puede encontrarle «lo propiamente americano» (31). Cada vez que escribe de Poe parece estar replicando los efectos de sus lecturas adolescentes de Darío, esas mismas que, a su vuelta del viaje a California, lo llevarán a plantearle los reclamos de la «Oda» que le dirige en 1927, a sus 21 años. En síntesis: la poética de Poe no lo habilita para proyectar una poética continental que implique una ruptura con la tradición europea, orientación definitiva que buscará, sin cesar, en la poesía modernista norteamericana como proyecto particular de su «destino-de-traducción»: proponer alternativas a la hegemonía dariana y al predominio de la biblioteca poética francesa en la literatura nicaragüense. El malentendido que cuenta en la crónica de su infancia es, por tanto, estratégico y programático: cuando lee a Poe —en realidad cuando lee la traducción de «El cuervo» de Poe de Pérez Bonalde—, afirma «creí que era francés» (27).

Quien recuerda que el adolescente había quedado impactado por la lectura de Whitman es el traductor que Urtecho ya es en 1962: a diferencia del adolescente, este sabe ahora que entonces no había leído a Whitman, sino una traducción de Whitman. El adolescente no decide por tanto su viaje a los Estados Unidos a los 18 años por Whitman; lo decide por una traducción. Es por tanto un lector de traducciones quien escribe: «Descubrí a Whitman en no recuerdo qué cantos suyos, traducidos por Amado Nervo, y la impresión que me produjeron...» (31). En su formación, el traductor no solo aprende una lengua al traducir; aprende además que una traducción tiene una entidad textual particular y que por tanto requiere también un estatuto propio de lectura. Por eso no es el niño ni el adolescente, sino el ya traductor quien escribe: «A pesar de ser Nervo lo menos "whitmaniano" posible, los tres o cuatro cantos por él traducidos, comunicaban una amplitud de proporciones continentales» (31). ¿Qué leer en este pasaje? En principio, la función necesaria de la traducción en su capacidad para expandir y sostener, benjaminianamente, la supervivencia de una obra. Para el adolescente Urtecho (cuyo inglés aprendido de una niñera negra y ya olvidado 
no le permitía leer «el original») es una traducción, por defectuosa que sea, la que lo habilita a empezar a «descubrir» no solo una literatura sino, inclusive, su propio «destino-de-traducción». La mención explícita tanto a Nervo y a Vassier como traductores de Whitman, así como a Pérez Bonalde como traductor de Poe, muestran una voluntad de volver visible la invisibilidad y lateralidad histórica del traductor: es él mismo quien está volviendo visible, oblicuamente, su propia labor desarrollada para entonces durante 35 años.

Pero ese reconocimiento repone simultánea y necesariamente cómo el traductor, lector de traducciones, es también crítico de traducciones: las versiones que hace Nervo de Whitman son «poco whitmanianas»; las de Vasseur, «secas, descoloridas» (31). Esas consideraciones suponen una instancia que el inglés aún incipiente (o aún más incipiente) de su juventud no le permitía: ahí está la confrontación de aquellas traducciones leídas en su adolescencia con la lectura posterior del Whitman «original». La crítica implicada en la lectura de la traducción cumple una función prioritaria: al reconocer los problemas de las traducciones existentes, genera las condiciones para diseñar las estrategias de una nueva. El traductor Urtecho que critica las traducciones de Nervo y Vasseur es por supuesto el que ya ha intentado versionar en español un Whitman más "whitmaniano». ¿Cómo se forma entonces un traductor? Leyendo traducciones sin saber que lo son. ¿En qué momento esa formación ofrece una ocasión de inflexión? Cuando se advierte que una traducción es una traducción. ¿Qué implica la adquisición de esa conciencia? La crítica. ¿Adónde lleva esa crítica? A una traducción nueva.

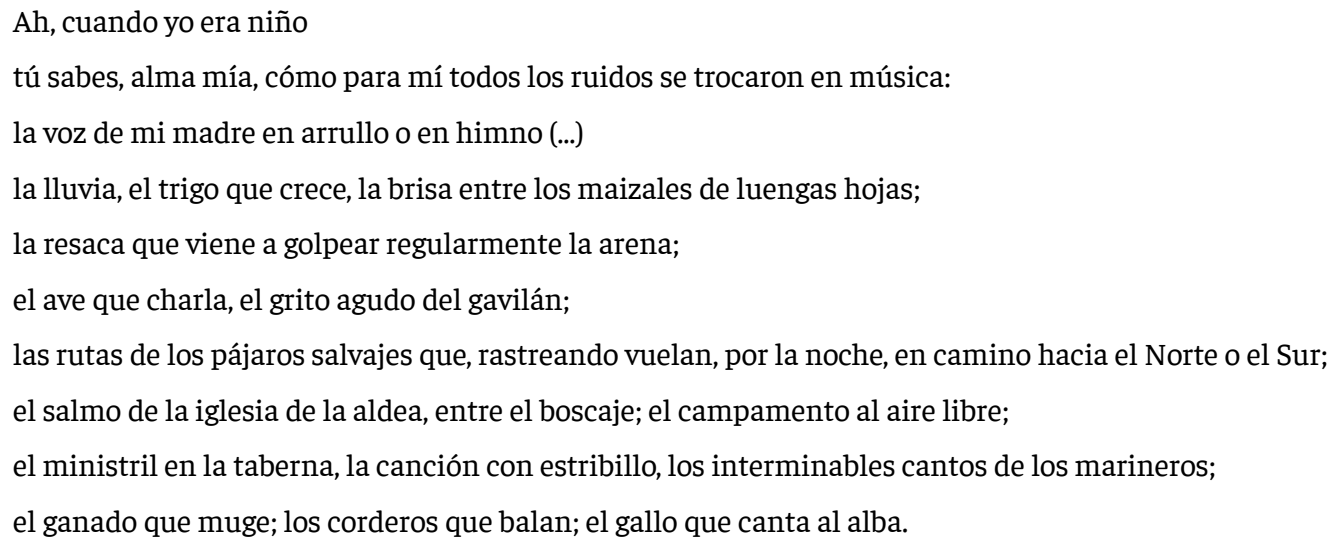

Aunque estaba leyendo la traducción que hace Amado Nervo del poema de Whitman «Proud music of the storm» (1920:228), el adolescente creía que estaba leyendo a Whitman. No es inverosímil imaginar que sea en esa escena de lectura, tramada por la falta de conciencia sobre el estatuto de su objeto, cuando Urtecho haya empezado a reconocer la dirección de un «destinode-traducción» más norteamericano que europeo. En la versión no está necesariamente el habla como modelo para la poesía, pero está el planteo de que hasta las hablas pueden devenir música, y está — sin duda — la música rara de un verso demasiado tendiente a la prosa. No hay que olvidar que la preferencia de Urtecho por Whitman con relación a Poe es también la preferencia por una poesía ya no más sostenida primariamente, a lo Verlaine, desde el nivel superior de lo musical. Porque, ¿cuál es la consecuencia de una poesía supeditada en primer lugar a la música, como la de Poe? Volverse intraducible. Así parecen demostrarlo, reflexiona Urtecho, las traducciones que encara el mismísimo Baudelaire, en una prosa resignada a segmentar el artificio mayor desde el que 
se sostiene. Whitman, por el contrario, es capaz de atravesar aún traducciones no whitmanianas. Apostar por Whitman es apostar por la traducibilidad como valor. Esa es la cualidad que busca Urtecho en la poesía «americana». Tal como el poema amadonerviano de Whitman lo muestra, no se trata de menos música, sino de otra música, capaz de incorporar al poema una serie innumerable de ruidos: la voz materna, la lluvia, el crecimiento del trigo, el viento entre los maizales, el ave que icharla!, los cantos de los marineros, el mugido del ganado, el balido del cordero, el canto de un gallo que anuncia otro comienzo. En efecto, Amado Nervo traduce «the twittering bird» como «el ave que charla»: ¿no se podría inclusive arriesgar más y afirmar que es ante ese verso, exactamente ante ese sintagma e inclusive ante ese verbo en particular, cuando el adolescente Urtecho encuentra el objeto de su «destino-de-traducción»? Ah, jexiste una poesía donde los pájaros ya no cantan!

No sería exacto decir que Urtecho no había conocido a ningún poeta modernista norteamericano antes de viajar a los Estados Unidos en 1924. Un año antes de su partida había leído a Robert Frost. En la crónica recuerda cuando su madre le muestra, con alegría e inclusive sorpresa, un artículo encomiástico sobre ese poeta en la revista... francesa Reveu des Deux Mondes: «estábamos encantados de que hubiera un poeta en los Estados Unidos, además de Longfellow» (1962:27). ¿En qué estribaba la alegría? «Ya no podría decirse que aquel país fuera... un país de banqueros y salchicheros millonarios, no más que práctico y mercantil, grosero y materialista, sin alma y sin poesía» (27). Por supuesto, el traductor recuerda que el adolescente leyó el artículo en francés «sin entenderlo del todo", aunque esa comprensión intermedia le haya alcanzado para retener a través de los años el nombre del poeta y recordar, incluso, que uno de los poemas se refería a «un macizo de flores» (27). Una vez más, por si fuera necesario, Urtecho presenta su crónica de infancia y adolescencia en clave programática. Porque no se trata solo de que haya leído a su primer poeta modernista norteamericano en francés (ien la lengua de los clásicos de Hachette, de Garnier y de los románticos como Lamartine!); la anécdota le brinda otra ocasión para insistir cómo su proyecto de traducción se inscribe decididamente en un pasaje de la poesía francesa a la norteamericana, presentando cómo, aún en esa época previa a su partida a los Estados Unidos, la autoridad literaria se seguía escribiendo en francés. Pero esa no es la única ironía de la anécdota: el Urtecho adolescente lee en francés justamente al poeta sobre cuyos dichos se sostendrán una y otra vez las justificaciones sobre la intraducibilidad de la poesía: «Poetry is what gets lost in translation» [«La poesía es lo que se pierde al traducirla»] $(2005,200)$. Sin duda el traductor Urtecho ya no se contará entre los militantes de esa sentencia; de hecho, aquella traducción no solo al francés sino en prosa de los versos de Frost (publicada en el tomo XVII de Revue des Deux Mondes, oct-sept de 1923, bajo el título «Poètes Américaines d'Aujourd'Hui: M. Robert Frost») le permite entrever algo a pesar de toda la pérdida involucrada. En definitiva, en aquel sintagma «macizo de flores» que mantiene en su recuerdo tal vez haya podido vislumbrar otra alternativa más para contrapesar la idea de poesía que circulaba en la casa de su abuelo, hecha de misterio y flores celestiales. Así, entonces, ya preparado para su «destino-de-traducción» - con el recuerdo y el olvido del inglés de la niñera negra, la lectura de Whitman y al menos un sintagma del modernismo en su memoria reciente-, un Urtecho adolescente sale de la casa tradicional, conservadora y colonial del abuelo para viajar a San Francisco, Estados Unidos. 


\section{Notas}

$1 \mathrm{Al}$ explicitar los materiales más adecuados para encarar una de las tareas de la «traductología», la de la «búsqueda del traductor», escribe Berman (2011:245): «Se puede pensar aquí en las "biografías" de traductores como Amyot, A. W. Schlegel, Armand Robin, en los análisis de destinos-de-traducción donde se aclararía la relación del traductor con la escritura, la lengua materna y las otras lenguas. Que yo sepa, esta analítica del traductor prácticamente no existe». En el póstumo Pour une critique des traductions (1995), dedicado a diseñar una

plataforma metodológica para el desarrollo de una «crítica de traducciones», Berman incluye también como etapa necesaria esa «búsqueda» particular, conceptualizando en este caso al traductor como aquel sujeto tomado por la «translation drive». Esa expresión —según explicita en una nota- es traducción del verbo utilizado por Schlegel en una carta a Novalis de 1797: «Übersetzungstrieb», el cual puede versionarse como «impulso a traducir». Escribe Berman: «es el impulso a traducir lo que hace de un traductor un traductor» (2009:58).

\section{Referencias bibliográficas}

Benjamin, W. (1971) [1923]. «La tarea del traductor». En Angelus novus. Barcelona: Edhasa, 127-143. Traducción de Héctor Murena.

Berman, A. (2011) [1989]. «La traducción y sus discursos». Mutatis mutandi, 4(2), 237-248. Traducción de John Jairo Gómez Montoya.

(1995). Pour une critique des traductions: John Donne. París: Gallimard.

(2009). [1995]. Towards a translation criticism: John Donne. Kent, Ohio: Kent State UP. Traducción de Francoise Massardier-Kenney.

Bourget, P. (1894). Outre-Mer(Notes sur L’Amérique). Tome Premier. París/New York: Alphonse Lemerre Editeur.

Coronel Urtecho, J. (1962). «El americanismo en la casa de mi abuelo». Revista Conservadora del Pensamiento Centroamericano, 5(23), 25-31.

(1927). «Los yanquis, poetas». El diario nicaragüense, 2 de octubre, 2.

(1949). Panorama y antología de la poesía norteamericana. Madrid: Seminario Problemas

Hispanoamericanos.

(1959) [1953]. Rápido tránsito. Al ritmo de Norteamérica. Madrid: Aguilar.

Coronel Urtecho, J. y Cardenal, E. (1951). El Lincoln de los poetas. Managua: El hilo azul.

(1963). Antología de la poesía norteamericana. Madrid: Aguilar.

(1983) [1979]. Antología de Ezra Pound. Madrid: Visor.

Frost, R. (2005) [1959]. Conversations on the craft of poetry with Cleanth Brooks and Robert Penn Warren. En D. Brown, A. Finch y M. Kumin (Eds.). Lofty dogmas: poets on poetics, Fayatteville: University of Arkansas Press, 196-203.

Nervo, A. (1920) [1904]. Música orgullosa de la tempestad. En Las voces. Lira heroica y otros poemas (Obras completas de Amado Nervo, v. III). Madrid: Biblioteca Nueva, 224-234.

«Poètes Américaines d'Aujourd'Hui: M. Robert Frost» (1923). Revue de deux mondes, tome XVII, SeptembreOctobre 1923, 185-210.

Rocha, L. (1993). «Prólogo conversado». En J. Coronel Urtecho. Pól-la d’ananta katánta paránta, dedójmia t'élson: imitaciones y traducciones. Managua: Nueva Nicaragua, 15-59. 